\title{
BIOANALYTICAL METHOD DEVELOPMENT AND VALIDATION OF TICAGRELOR BY RP-HPLC
}

\author{
DELMA DCRUZ, ANU BABU, EENA JOSHY, ANEESH T. P.*
}

\author{
School of Pharmacy, Amrita University, Amrita Vishwa Vidyapeetham, AIMS Health Sciences Campus, Kochi, India \\ Email: aneeshtp@aims.amrita.edu
}

Received: 12 Nov 2016, Revised and Accepted: 17 Apr 2017

\section{ABSTRACT}

Objective: The main purpose of this study was to develop a simple, precise, rapid and accurate RP-HPLC method for the quantitative determination of ticagrelor in human plasma.

Methods: The separation was accomplished by the isocratic method by utilizing phenomenex C18 column on a Shimadzu binary gradient liquid chromatography system furnished with LC-20AD solvent delivery system, SPD-20-A photo-diode array detector and $20 \mu \mathrm{l}$ loop volume in a rheodyne injector. The analyte was extracted by protein precipitation in the involvement of diethyl ether as a protein precipitator. The mobile phase was developed for the estimation of the drug in human plasma consists of acetonitrile and methanol in the ratio of 60:40\% v/v. Separation was done with a flow rate of $1 \mathrm{ml} / \mathrm{min}$ at a detection wavelength of $254 \mathrm{~nm}$.

Results: Retention time was found to be 4.503 min with a run time 10 min. Linearity shows in a range of $20-100 \mu \mathrm{g} / \mathrm{ml}$, with a correlation coefficient of 0.9992 respectively. Stability studies of ticagrelor in plasma were carried out by, short term stability, long term stability and bench top stability studies. Short term stability, long term stability and bench top stability of ticagrelor was carried out from 20 and $100 \mu \mathrm{g} / \mathrm{ml}$ concentration and $\%$ RSD was ascertained $0.12 \%$ and $0.08 \%, 0.18 \%$ and $0.15 \%, 1.19 \%$ and $1.30 \%$ respectively.

Conclusion: The outcomes were observed to be inside the knowledge of ICH guidelines. The prepared solution was injected in triplicate, and \% RSD was measured. Acquired results demonstrate that proposed strategy can be effortlessly and advantageously applied for routine examination of ticagrelor in human plasma.

Keywords: Bioanalytical method, Reverse phase HPLC, Ticagrelor, ICH

(C) 2017 The Authors. Published by Innovare Academic Sciences Pvt Ltd. This is an open access article under the CC BY license (http://creativecommons.org/licenses/by/4.0/) DOI: http://dx.doi.org/10.22159/ijap.2017v9i3.17452

\section{INTRODUCTION}

Bioanalysis is the strategy used to determine the concentration of drug and metabolites in the biological matrices like plasma, serum, cerebrospinal fluid, urine, saliva etc. Bioanalytical method and validation are utilized to build up, that a quantitative analytical method can be connected for the biochemical process. Validation involves documentation of laboratory investigations that the method is suitable and reliable for the intended applications [1]. It is utilized for the assessment of bioavailability and bioequivalence studies, quantitative evaluation of drug and metabolites, new drug development, clinical pharmacokinetics, research process and therapeutic drug monitoring. Bioanalytical techniques are constantly undergoing changes and improvements that they are the cutting edge of technology [2].

Ticagrelor is an anti-platelet drug which is chemically $(1 \mathrm{~S}, 2 \mathrm{~S}, 3 \mathrm{R}, 5 \mathrm{~S})-3-[7-$ [(1R,2S)-2-(3,4-Difluorophenyl)cyclopropylamino]-5-(propyl thiol)-3H$[1,2,3]$ triazolo[4,5-d]pyrimidin-3-yl]-5-hydroxyethoxy)cyclopentane1,2-diol. It blocks adenosine diphosphate (ADP) receptors of subtype P2Y12 in contrast to other antiplatelet drugs, ticagrelor has a binding site making it an allosteric antagonist and the blockage is reversible [3]. It inhibits platelet aggregation and thrombus formation in atherosclerotic disease by preventing the activation of platelet [4]

The present study portrays the improvement and acceptance of a delicate, particular, fast, straight forward and cost effective HPLC bioanalytical strategy for ticagrelor in human plasma as indicated by ICH Q2 (R1) and EMA guidelines [5, 6]. The objective of validation of bioanalytical procedure is to demonstrate that it is suitable for the intended purpose and it will be beneficial for the researchers.

\section{MATERIALS AND METHODS}

\section{Chemicals and reagents}

The active pharmaceutical ingredients were benevolently acquired from swapnaroop drugs and pharmaceuticals, Maharashtra, India. The pharmaceutical formulations were purchased from the local market which contains ticagrelor $90 \mathrm{mg}$. required chemicals, reagents, acetonitrile, methanol used are of HPLC grade.

\section{Optimization of chromatographic conditions}

Separation was done by using phenomenex C18 $(250 \times 4.6 \mathrm{~mm}, 5$ $\mu \mathrm{m})$ column on a shimadzu liquid chromatographic system with LC20AD solvent delivery system, SPD-20A photodiode array detector and rheodyne injector with $20 \mu \mathrm{l}$ loop volume. Mobile phase consists of a mixture of acetonitrile and methanol in the ratio of $60 \% \mathrm{v} / \mathrm{v}$ in pump A and $40 \% \mathrm{v} / \mathrm{v}$ in pump B was found to be the most suitable mobile phase for ideal chromatographic separation of ticagrelor. It was pumped with a flow rate of $1.0 \mathrm{ml} / \mathrm{min}$ through the column. The column was equilibrated by pumping the mobile phase for $30 \mathrm{~min}$ prior to the injection of the drug solution. The detection of the drug was monitored at $254 \mathrm{~nm}$. The run time was set at $10 \mathrm{~min}$.

\section{Preparation of stock solution}

Blood samples were collected and centrifuged at 10,000 rpm for 2 min. The plasma thus obtained were separated and stored. $1 \mathrm{mg}$ of standard ticagrelor was weighed accurately and spiked into $1 \mathrm{ml}$ plasma. After centrifugation, subsequent aliquots of $20-100 \mu \mathrm{g} / \mathrm{ml}$ concentrations were prepared by diluting with $100 \mu \mathrm{l}$ of diethyl ether and the mobile phase and clear supernatant liquid of each concentration were injected for analysis and chromatogram were taken [7]. The chromatogram is shown in fig. 1.

\section{Calibration curve}

The calibration plots were constructed by plotting peak area against respective concentrations. Linearity was obtained by analysis of serially diluted sample in the range of $20,40,60,80,100 \mu \mathrm{g} / \mathrm{ml}$. Calibration graph is shown in fig. 2.

\section{Validation of the method}

The developed method was validated by evaluating linearity, precision, accuracy and specificity which were done in acceptance of ICH [8] and EMA guidelines. 


\section{Linearity}

Linearity was studied by standard solutions at different concentration levels. The linearity range for ticagrelor was found to be $20-100 \mu \mathrm{g} / \mathrm{ml}$.
The regression equation was found to be $y=35798 x-9772.3$ with a coefficient of correlation $\left(\mathrm{r}^{2}\right)$ 0.9992. The correlation coefficient for linearity is 0.9999 . Since the result is approximately close to the true value, the method is indicated as highly significant.

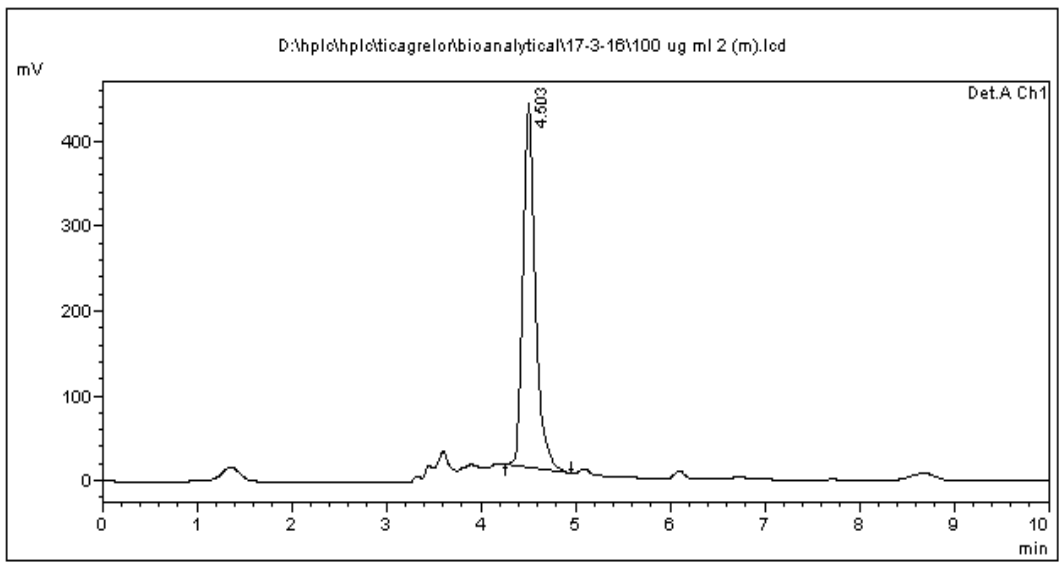

Fig. 1: Chromatogram of ticagrelor in human plasma $100 \mu \mathrm{g} / \mathrm{ml}$

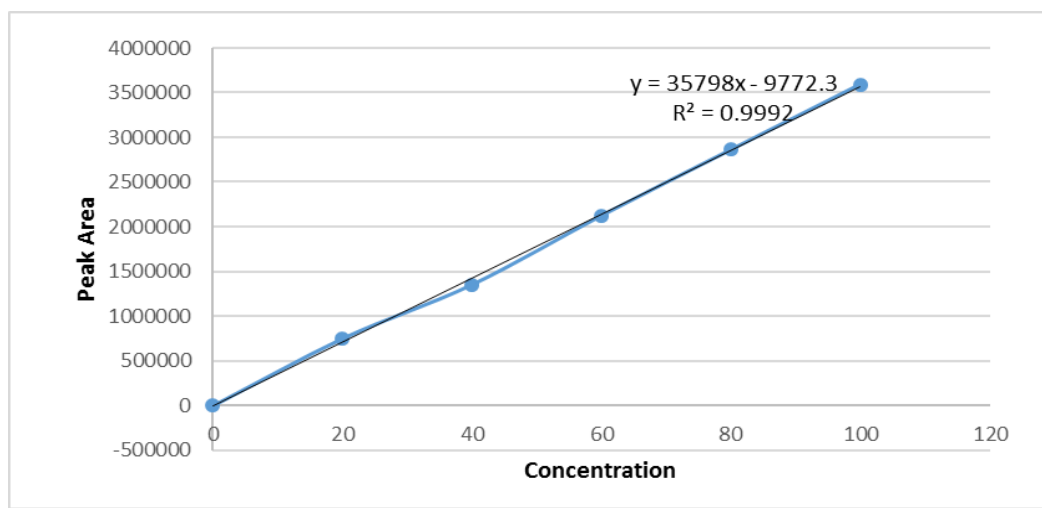

Fig. 2: Calibration curve of ticagrelor in human plasma

Table 1: Linearity of ticagrelor in human plasma

\begin{tabular}{ll}
\hline Concentration $(\boldsymbol{\mu g} / \mathbf{m l})$ & Area \\
\hline 20 & 750690 \\
40 & 1352882 \\
60 & 2124666 \\
80 & 2861693 \\
100 & 3590697 \\
\hline
\end{tabular}

\section{Precision}

Precision was determined by carrying three replicates of concentration $40 \mu \mathrm{g} / \mathrm{ml}$ and performed intraday (within a day) and interday (day to day) studies. The percentage relative standard deviation (\%RSD) was found to be less than 1\%. For Intraday precision study, evaluation was carried out by injecting a standard solution at various time intervals and \%RSD of Ticagrelor was found to be $0.56 \%$ and $0.51 \%$ shown in table 2 where inter-day precision was carried out in consecutive days with \%RSD of $0.76 \%$ and $0.81 \%$ shown in (table 3). The \%RSD can be reached up to $2 \%$

Since the outcome is less than $1 \%$ it was found to be satisfactory, which indicates method is precise [9].

Table 2: Intraday study of ticagrelor in human plasma

\begin{tabular}{llll}
\hline Days & Standard concentration & Peak area \\
\hline $1^{\text {st }}$ day & 40 & Morning & \\
& & 320815 & \\
& & 3212878 & 3202864 \\
& & 3218728 & \\
& & 3238005 & \\
& MEAN & 3217899.33 & 3219199 \\
& STD DEV & 18124.41 & 16575.51 \\
\end{tabular}


Table 3: Interday study of ticagrelor in human plasma

\begin{tabular}{lll}
\hline Standard concentration & Peak area & \\
\hline 40 & Day 1 & Day 2 \\
& 1730789 \\
& 1730997 & 1742415 \\
MEAN & 1742154 & 1758924 \\
STD DEV & 1757249 & 1744042.66 \\
\% RSD & 1743466.66 & 14137.94 \\
\hline
\end{tabular}

\section{Limit of detection (LOD)}

LOD was determined by standard deviation method and slope of the calibration plot by using the formula $3.3^{*} \sigma / \mathrm{S}$. It was observed to be $0.382 \mu \mathrm{g} / \mathrm{ml}$. since the observed concentration is low, the method is sufficiently sensitive.

\section{Limit of quantification (LOQ)}

LOD was determined by standard deviation method and slope of the calibration plot by using the formula $10^{*} \sigma / \mathrm{S}$. It was observed to be $1.158 \mu \mathrm{g} / \mathrm{ml}$. As the amount of analyte was found to be less, we can estimate the drug at very low concentration.

\section{Stability of ticagrelor in human plasma}

\section{Short term stability}

Short term stability was determined by storing concentration of 20 and $100 \mu \mathrm{g} / \mathrm{ml}$ over a period of $6 \mathrm{~h}$ at room temperature. Stability was determined by performing three replicates and calculated $\%$
RSD. It was found to be $0.12 \%$ and $0.08 \%$ respectively, shown in table 4 as it is less than $1 \%$ the method is said to be stable.

\section{Long term stability}

Long-term stability was determined by storing concentration of 20 and $100 \mu \mathrm{g} / \mathrm{ml}$ over a period of $10 \mathrm{~d}$ at room temperature. Stability was determined by calculating the \% RSD. It was found to be $0.18 \%$ and $0.15 \%$ respectively, as it is less than $1 \%$ the method can said to be stable.

\section{Bench top stability}

Three replicates of the lowest and higher concentration were determined. The samples were assessed after keeping at room temperature (bench top) against freshly prepared concentrations and \%RSD was calculated [10]. It was found to be $1.19 \%$ and $1.30 \%$ respectively. The \%RSD can be reached up to $2 \%$. Since the result is less than $2 \%$ values were found to be satisfactory, which indicates method is stable.

Table 4: Short-term stability of ticagrelor

\begin{tabular}{lllll}
\hline Concentration & Peak area & Mean & Standard deviation & \%RSD \\
\hline 20 & 1929188 & 1927512 & 2385.189 & $0.12 \%$ \\
& 1922566 & & & \\
& 1924781 & & & \\
100 & 5491030 & 5495356 & & \\
& 5495513 & & & \\
& 5499524 & & & \\
\hline
\end{tabular}

Table 5: Long-term stability of ticagrelor

\begin{tabular}{lllll}
\hline Concentration & Peak area & Mean & Standard deviation & \%RSD \\
\hline 20 & 1152882 & 1153686 & 2065.306 & $0.18 \%$ \\
& 1156032 & & & \\
& 1152143 & & & \\
100 & 4891697 & 489020 & & \\
& 4882466 & & & \\
& 4897096 & & & \\
\hline
\end{tabular}

Table 6: Bench top stability of ticagrelor

\begin{tabular}{llll}
\hline Concentration & Old & Fresh & \% RSD \\
\hline 20 & 1077812 & 1096036 & $1.19 \%$ \\
100 & 5342790 & 5442090 & $1.30 \%$ \\
\hline
\end{tabular}

\section{CONCLUSION}

The developed RP-HPLC method was found to be simple, precise, accurate and sensitive for the estimation of Ticagrelor in human plasma. Validation of results according to ICH and EMA carried out revealed high accuracy and good precision. The RSD for every one of the parameters are observed to be short of what one, which shows the legitimacy of the technique is reasonably fine. A mixture of acetonitrile and methanol mobile phase ratio of $60 \% \mathrm{v} / \mathrm{v}$ in pump $\mathrm{A}$ and $40 \% \mathrm{v} / \mathrm{v}$ in pump B at a flow rate of $1 \mathrm{ml} / \mathrm{min}$. The wavelength was found to be $254 \mathrm{~nm}$ in $\mathrm{U} . \mathrm{V}$ spectroscopy. Retention time was found to be $4.503 \mathrm{~min}$ with a run time $10 \mathrm{~min} .20-100 \mu \mathrm{g} / \mathrm{ml}$ concentration of ticagrelor shows linearity with a correlation coefficient of 0.9992 . Thus we can conclude that this method can be easily and conveniently adopted for the quality control analysis of ticagrelor in human plasma.

\section{CONFLICTS OF INTERESTS}

Declare none

\section{REFERENCES}

1. Kirthi R, Shanmugam R, Shanti Prathyusha M, Jamal Basha D. A review on bioanalytical method development and validation by RP-HPLC. J Glob Trends Pharm Sci 2014;5:2265-71.

2. Goel D. Ticagrelor. The first approved reversible oral antiplatelet agent. Int J Appl Basic Med Res 2013;3:19-21.

3. RaeAnne, Benjamin Chavez. Ticagrelor (Brilinta), An antiplatelet drug for acute coronary syndrome. P and T; 2012. p. 562-8.

4. Khaja Pasha, Asgar Ali, Shahana Bana, Syeda Humair. Reverse phase-HPLC method for the analysis of tinidazole in the 
pharmaceutical dosage form and bulk drug. Int J Pharm Pharm Sci 2010;2:47-52.

5. Guideline on Bioanalytical method validation: European Medicines Agencies; 2011.

6. Sonawane LV, Poul BN, Usnale SV. Bioanalytical method validation and its pharmaceutical application a review. Pharm Anal Acta 2014;5:1-7.

7. Megha G Gore, Pratap S Dabhade. RP-HPLC method development and validation of azelnidipine. Int J Pharm Sci Res 2016;5:111-4.

8. Pushpa Latha E, Sailaja B. Bioanalytical method development and validation by HPLC: a review. J Appl Pharm 2014;1:1-9.
9. Choudary S, Saahil Arora, Tanvil Sharma. Bioanalytical method development and validation of ibuprofen using RP-HPLC. Pharm Tech Res 2012;2:312-21.

10. Rutvik Pandya H, Rajeshwari Rathod, Dilip G. Development and validation for simultaneous determination of linagliptin and metformin drugs in human plasma by the RP-HPLC method. Pharmacophore 2014;6:202-18.

\section{How to cite this article}

- Delma D’cruz, Anu Babu, Eena Joshy, Aneesh TP. Bioanalytica method development and validation of ticagrelor by RP-HPLC. Int J Appl Pharm 2017;9(3):51-54 